\title{
Erratum: Kinetic theory of shear thickening for a moderately dense gas-solid suspension: From discontinuous thickening to continuous thickening [Phys. Rev. E 96, 042903 (2017)]
}

Hisao Hayakawa $『$, Satoshi Takada, and Vicente Garzó

(Received 3 June 2020; published 30 June 2020)

DOI: 10.1103/PhysRevE.101.069904

We have realized that there are several typographical errors and mistakes in this paper which cause significant changes. Euation (3) should read

$$
\left\langle\xi_{i}(t)\right\rangle=0, \quad\left\langle\xi_{i, \alpha}(t) \xi_{j, \beta}\left(t^{\prime}\right)\right\rangle=\frac{2 \zeta T_{\mathrm{ex}}}{m} \delta_{i j} \delta_{\alpha \beta} \delta\left(t-t^{\prime}\right) .
$$

Equation (17) in the paper should read

$$
T=\frac{m}{d n} \int d \boldsymbol{V} \boldsymbol{V}^{2} f(\boldsymbol{V})
$$

The expression of Eq. (32) of this paper is not for $\bar{\Lambda}_{\alpha \beta}^{E}$ but for $\Lambda_{\alpha \beta}^{E}$ as

$$
\begin{aligned}
\Lambda_{\alpha \beta}^{E}= & g_{0} n T\left\{\nu \Pi_{\alpha \beta}^{k}+\lambda \delta_{\alpha \beta}-\frac{2^{d-2}}{(d+2)(d+4)} \varphi(1+e) \dot{\gamma}\left[(d+4)(1-3 e)\left(\delta_{\alpha x} \delta_{\beta y}+\delta_{\alpha y} \delta_{\beta x}\right)\right.\right. \\
& \left.\left.+2(d+1-3 e)\left(\Pi_{\alpha x}^{k} \delta_{\beta y}+\Pi_{\alpha y}^{k} \delta_{\beta x}+\Pi_{\beta x}^{k} \delta_{\alpha y}+\Pi_{\beta y}^{k} \delta_{\alpha x}\right)-6(1+e) \delta_{\alpha \beta} \Pi_{x y}^{k}\right]\right\} .
\end{aligned}
$$

The above error yields substantial changes in expressions displayed in the paper. In particular, Appendix $C$ of this paper should be largely modified. Since there are so many changes in Appendix C (as well as some typographical errors), we have rewritten it as Appendix B in Ref. [1]. The summary of the changes in Appendix C of the paper is as follows. (i) The left-hand side of Eq. (C1) should read $\Lambda_{\alpha \beta}^{E}$. (ii) $\left|\boldsymbol{v}_{12} \cdot \hat{\boldsymbol{\sigma}}\right|$ in the first line of Eq. (C2) should read $\left(\boldsymbol{v}_{12} \cdot \hat{\boldsymbol{\sigma}}\right)$. (iii) $\partial f\left(\boldsymbol{V}_{2}^{\prime \prime}\right) / \partial V_{2, x}$ in the first line of Eq. (C5) should read $\partial f\left(\boldsymbol{V}_{2}^{\prime \prime}\right) / \partial V_{2, x}^{\prime \prime}$. (iv) We should remove one integral with respect to $\boldsymbol{V}_{2}$ in the last expression of Eq. (C5). (v) $V_{1, \alpha}^{\prime} V_{2, \beta}^{\prime}-V_{1, \alpha} V_{2, \beta}$ in the last term of Eq. (C5) should be $V_{1, \alpha}^{\prime} V_{1, \beta}^{\prime}-V_{1, \alpha} V_{1, \beta}$. (vi) We should replace $\Pi_{\mu \nu}$ in Eqs. (C4), (C11), (C20), and right after Eq. (C24) by $\Pi_{\mu \nu}^{k}$. (vii) Similarly, $\Pi_{\mu \nu}$ at the beginning of Eq. (C28) should read $\Pi_{\mu \nu}^{k}$. (viii) $V_{G, \alpha} \hat{\sigma}_{\alpha}$ in Eq. (C31) should read $V_{G, \alpha} \hat{\sigma}_{\beta}+V_{G, \beta} \hat{\sigma}_{\alpha}$. (ix) In Eq. (C32), we miss $G_{\beta} \hat{\sigma}_{\alpha}$ in addition to $G_{\alpha} \hat{\sigma}_{\beta}$ in the first line. (x) We should remove $m /(2 T)$ and add $\dot{\gamma}^{\prime} \hat{\sigma}_{y}^{2} \delta_{\alpha x} \delta_{\beta x} / 4$ in the brackets including $2 G_{\alpha} G_{\beta}+g_{\alpha} g_{\beta} / 2$ in the second line of Eq. (C32).

With the change in Eq. (32) of the paper, the set of coupled differential Eqs. (35)-(38) of the paper becomes independent of the collisional contribution $P_{\alpha \beta}^{c}$. Thus, we can determine $T, \Delta T$, and $\delta T$ from the closed set of equations as

$$
\begin{gathered}
\frac{\partial}{\partial t} T=-\frac{2 \dot{\gamma}}{d n} \mathcal{C}_{d} P_{x y}^{k}+2 \zeta\left(T_{\mathrm{ex}}-T\right)-g_{0} \lambda T \\
\frac{\partial}{\partial t} \Delta T=-\frac{2}{n} \dot{\gamma} P_{x y}^{k}-\left(\nu g_{0}+2 \zeta\right) \Delta T \\
\frac{\partial}{\partial t} \delta T=-\frac{2}{n} \dot{\gamma} \mathcal{E}_{d} P_{x y}^{k}-\left(\nu g_{0}+2 \zeta\right) \delta T \\
\frac{\partial}{\partial t} P_{x y}^{k}=\dot{\gamma} n\left(\frac{d-1}{d} \mathcal{D}_{d} \Delta T-\frac{d-2}{d} \mathcal{E}_{d} \delta T-\mathcal{C}_{d} T\right)-\left(\nu g_{0}+2 \zeta\right) P_{x y}^{k},
\end{gathered}
$$

where the (dimensionless) quantities $\mathcal{C}_{d}, \mathcal{E}_{d}$, and $\mathcal{D}_{d}$ are given by Eqs. (39)-(41), respectively, of the paper.

In the steady state, the constitutive equations (46)-(49) of the paper for the dimensionless rheological properties should read

$$
\begin{gathered}
-\frac{2 \dot{\gamma}^{*}}{d R} \mathcal{C}_{d} \Pi_{x y}^{k}=g_{0} \sqrt{\theta} \lambda^{*}+2\left(1-\theta^{-1}\right), \\
-\frac{2 \dot{\gamma}^{*}}{R} \Pi_{x y}^{k}=\left(2+g_{0} \sqrt{\theta} v^{*}\right) \frac{\Delta \theta}{\theta},
\end{gathered}
$$




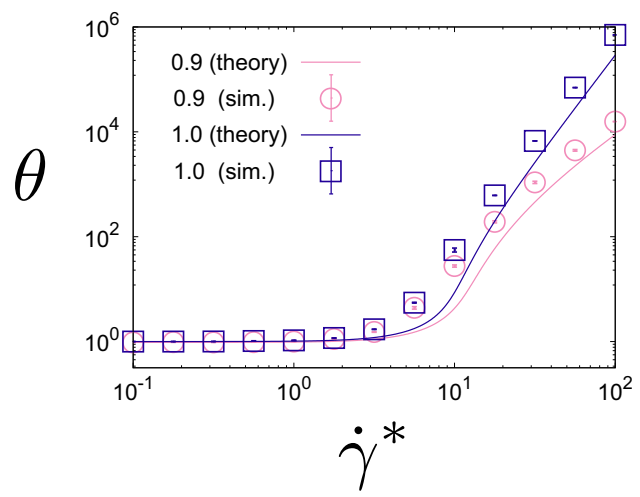

(a)

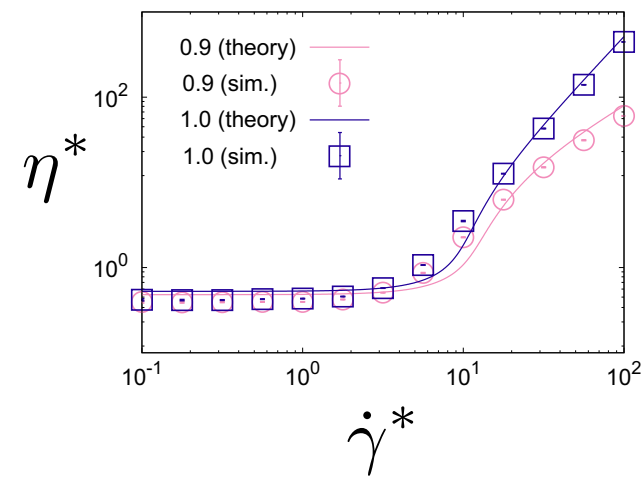

(b)

FIG. 5. Plots of (a) $\theta$ and (b) $\eta^{*}$ against $\dot{\gamma}^{*}$ for $\varphi=0.20$ and two values of the restitution coefficient $(e=0.9$ and $e=1)$. The lines refer to theoretical results whereas the symbols correspond to simulation results.

$$
\begin{gathered}
-\frac{2 \dot{\gamma}^{*}}{R} \mathcal{E}_{d} \Pi_{x y}^{k}=\left(2+g_{0} \sqrt{\theta} v^{*}\right) \frac{\delta \theta}{\theta}, \\
\left(2+v^{*} g_{0} \sqrt{\theta}\right) \Pi_{x y}^{k}=\frac{\dot{\gamma}^{*}}{R}\left(\frac{d-1}{d} \mathcal{D}_{d} \frac{\Delta \theta}{\theta}-\frac{d-2}{d} \mathcal{E}_{d} \frac{\delta \theta}{\theta}-\mathcal{C}_{d}\right),
\end{gathered}
$$

where the dimensionless shear rate $\dot{\gamma}^{*}$ is introduced in Eq. (44) in the paper.

Thanks to the absence of $P_{\alpha \beta}^{c}$ in Eqs. (35)-(38) in this Erratum, the steady solution of these equations corresponding to Eqs. (50)-(52) in the paper can be simplified as

$$
\begin{gathered}
\Pi_{x y}^{k}=-\frac{d R}{2 \dot{\gamma}^{*} \mathcal{C}_{d}}\left[g_{0} \sqrt{\theta} \lambda^{*}+2\left(1-\theta^{-1}\right)\right], \\
\frac{\Delta \theta}{\theta}=\frac{d}{\mathcal{C}_{d}} \frac{g_{0} \sqrt{\theta} \lambda^{*}+2\left(1-\theta^{-1}\right)}{2+g_{0} \sqrt{\theta} \nu^{*}}, \\
\frac{\delta \theta}{\theta}=\frac{d \mathcal{E}_{d}}{\mathcal{C}_{d}} \frac{g_{0} \sqrt{\theta} \lambda^{*}+2\left(1-\theta^{-1}\right)}{2+g_{0} \sqrt{\theta} \nu^{*}}
\end{gathered}
$$

As a result, to obtain $\dot{\gamma}^{*}$ in terms of $\theta$, we do not need to expand $\dot{\gamma}^{*}$ in powers of the parameter $\tau_{T}$ [defined in Eq. (44)]. The closed form of $\dot{\gamma}^{*}$ is now given by

$$
\dot{\gamma}^{*}=R \sqrt{\frac{-d\left[g_{0} \sqrt{\theta} \lambda^{*}+2\left(1-\theta^{-1}\right)\right]\left(2+g_{0} \sqrt{\theta} \nu^{*}\right)}{2 \mathcal{C}_{d} \mathcal{F}_{d}(\theta)}},
$$

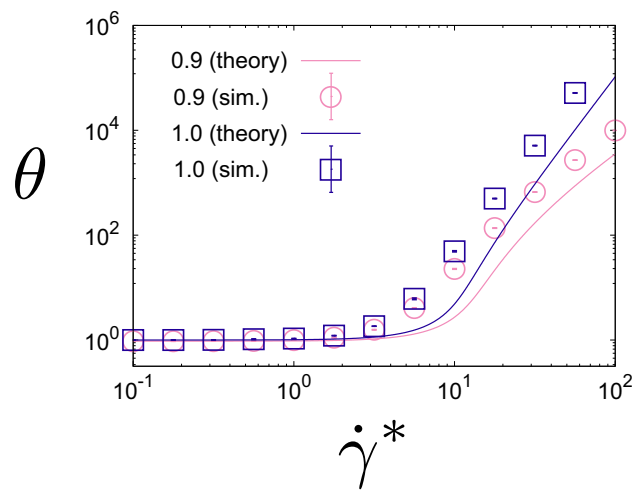

(a)

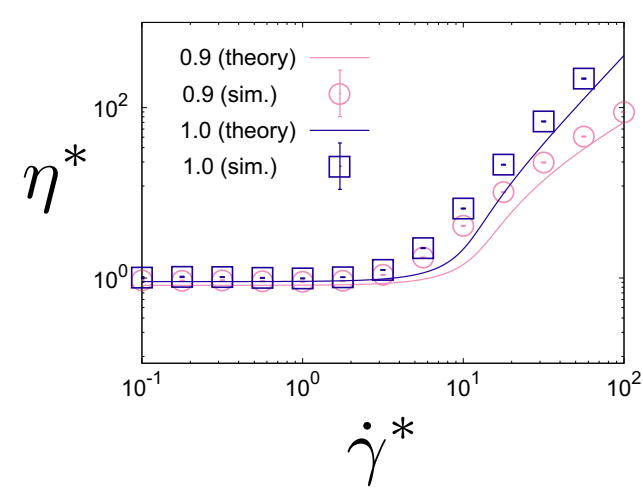

(b)

FIG. 6. Plots of (a) $\theta$ and (b) $\eta^{*}$ against $\dot{\gamma}^{*}$ for $\varphi=0.30$ and two values of the restitution coefficient $(e=0.9$ and $e=1)$. The lines refer to theoretical results whereas the symbols correspond to simulation results. 
where $\mathcal{F}_{d}(\theta)$ is

$$
\mathcal{F}_{d}(\theta)=\frac{d-1}{d} \mathcal{D}_{d} \frac{\Delta \theta}{\theta}-\frac{d-2}{d} \mathcal{E}_{d} \frac{\delta \theta}{\theta}-\mathcal{C}_{d}=\frac{(d-1) \mathcal{D}_{d}-(d-2) \mathcal{E}_{d}^{2}}{\mathcal{C}_{d}} \frac{g_{0} \sqrt{\theta} \lambda^{*}+2\left(1-\theta^{-1}\right)}{2+g_{0} \sqrt{\theta} \nu^{*}}-\mathcal{C}_{d} .
$$

Equation (67) of the paper is now more precisely computed by employing Eq. (A) for $\dot{\gamma}^{*}$.

We also note that the simulations presented in the paper contained some coding errors in addition to the changes in analytic expressions. Therefore, the comparison carried out in Figs. $2-11$ between simulations and theoretical results should be changed.

Due to the limitation of length in this Erratum, we only present two figures for a three-dimensional system with $\varphi=0.2$ and 0.3. As results of fixing all errors, we found that Figs. 5 and 6 in the paper should be replaced by Figs. 5 and 6, respectively, of the present Erratum.

Important changes in the revised theory from this paper are that: (i) The theoretical expression of $\theta$ does not depend on $P_{\alpha \beta}^{c}$, and (ii) the theoretical results of $\eta^{*}$ are improved when the shear-rate dependence of $P_{\alpha \beta}^{c}$ is accounted for. Nevertheless, as summarized in the paper, the comparison between the revised theory and the simulation indicates that the theoretical predictions for the kinetic temperature and the other rheological quantities (the viscosity, the stress ratio, and the normal stress differences) reasonably agree with simulations for densities $\varphi \lesssim 0.3$ and not quite strong inelasticity. In addition, the main conclusions of the paper are not affected by the revised theory since the present results still show a transition from discontinuous changes in $\theta$ and $\eta^{*}$ for dilute suspensions to continuous changes as the density increases.

We thank A. Santos for his indication of our mistakes and fruitful discussion to fix them.

[1] S. Takada, H. Hayakawa, A. Santos, and V. Garzó, arXiv:2005.05969v1. 